\title{
A Rare Case of Chronic Abruption-oligohydramnios Sequence at 16 Weeks Pregnancy with Compensated Severe Anemia
}

\author{
Kanika Chopra
}

\begin{abstract}
Aim: The aim of this article is to report a rare case of chronic abruption-oligohydramnios sequence (CAOS).

Background: Abruptio placentae is usually an acute event needing emergent measures for management, but may present with varied clinical features.
\end{abstract}

Case report: We describe a rare case of a 20 -year-old primigravida at 16 weeks of gestation who presented with complaints of mild pain abdomen off and on for 1 month and minimal bleeding per vaginum for a day. Her vital signs were stable, but there was marked pallor. Obstetric examination revealed uterus corresponding to 20 to 22 weeks of gestation with raised basal tone. Ultrasound findings showed a dead fetus corresponding to 16 weeks of gestation with a large retroplacental collection of $12.5 \times 7 \mathrm{~cm}$. The patient was given three units of blood transfusion after which the patient went into spontaneous labor and delivered uneventfully 23 hours after admission.

Conclusion: Recognition of chronic abruption and prompt intervention in our case led to a favorable maternal outcome.

Clinical significance: The diagnosis of chronic abruption should be kept in mind in patients presenting with pain abdomen even in early pregnancy.

Keywords: Chronic abruption, Chronic abruption-oligohydramnios sequence, Oligohydramnios, Second trimester, Severe anemia.

How to cite this article: Chopra K. A Rare Case of Chronic Abruption-oligohydramnios Sequence at 16 Weeks Pregnancy with Compensated Severe Anemia. World J Anemia 2017;1(2):65-67.

Source of support: Nil

Conflict of interest: None

\section{BACKGROUND}

Premature separation of placenta before birth has a wide spectrum of clinical presentation and occurs in 0.5 to $1.5 \%$

\section{Senior Resident}

Department of Obstetrics and Gynecology, Lady Hardinge Medical College, Ghaziabad, Uttar Pradesh, India

Corresponding Author: Kanika Chopra, Senior Resident Department of Obstetrics and Gynecology, Lady Hardinge Medical College, Ghaziabad, Uttar Pradesh, India, Phone: +919958121123, e-mail: kank2kanu@yahoo.co.in of all pregnancies. ${ }^{1}$ Minor degrees of placental abruption will lead to minimal signs and symptoms in the form of slight vaginal bleeding, vague abdominal pain, and preterm labor pains. More extensive placental separation leads to fetal distress and even fetal demise. In worse scenarios, it may be associated with maternal decompensation in the form of hypotensive shock, postpartum hemorrhage leading to anemia, blood transfusions, disseminated intravascular coagulation, acute renal failure, acute respiratory distress syndrome, and even maternal death. Placental abruption is most frequently observed during the 24th to 26th weeks of pregnancy, decreasing in frequency thereafter. Onset before 20 weeks of pregnancy is very rare. ${ }^{2}$ Similarly, abruption as the end result of chronic inflammatory process starting early in pregnancy is in itself a rare entity. Here, we report a rare case of chronic abruption presenting with 16 weeks of gestation associated with severe anemia.

\section{CASE REPORT}

The patient was a 20-year-old, unbooked primigravida who presented at 16 weeks of gestation with complaints of mild pain abdomen for 1 month and minimal bleeding per vaginum for 1 day. There was history of progressively increasing exertional dyspnea and easy fatigability for 1 month. There was no history of trauma or leaking per vaginum. There was no significant past, personal, or family history. On examination, the patient had severe pallor. Her pulse rate was 114 beats per minute (regular and good in volume), blood pressure was $100 / 60 \mathrm{~mm} \mathrm{Hg}$, respiratory rate was 20 per minute, and saturation was $100 \%$ on room air. Her jugular venous pressure was normal and chest was bilaterally clear with no added sounds. On per abdominal examination, uterus was 20 to 22 weeks with raised basal tone and on per speculum examination, no active bleeding was present.

Ultrasound was done and it revealed a dead fetus of 16 weeks gestation, with reduced liquor associated with a large retroplacental hematoma of $12.7 \times 7 \mathrm{~cm}$. Her initial laboratory parameters were hemoglobin $2.2 \mathrm{gm} \%$, platelet count 1.54 lacs per microliter, and total leukocyte count $20,500 / \mu \mathrm{L}$. Her prothrombin time was 11.8 , partial prothrombin time was 22.3 , international normalized 


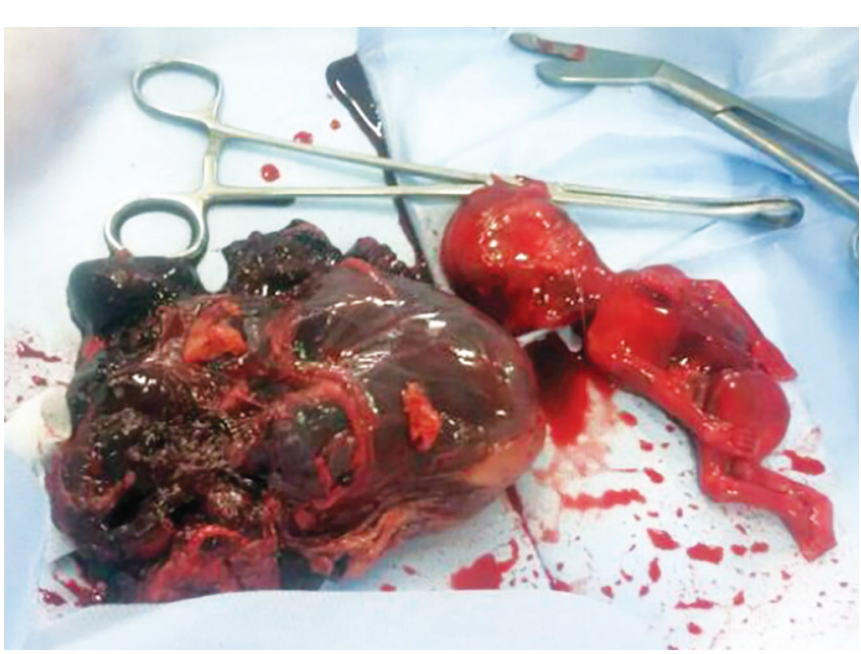

Fig. 1: Dead fetus and placenta with retroplacental clot

ratio was 1.07 , blood urea and serum creatinine were 40 and 0.7 respectively, and liver function tests were within normal limit.

So a diagnosis of primigravida with 16 weeks of gestation with intrauterine fetal demise with CAOS with compensated severe anemia was made based on clinical and ultrasound findings.

She was given three units of blood transfusion and her vital signs were monitored. Patient went into spontaneous labor and delivered a male dead fetus weighing $100 \mathrm{gm}$ with a retroplacental blood clot of $500 \mathrm{gm}$ (Fig. 1) after 23 hours of admission. Placenta was sent for histopathology and revealed hemorrhage adjacent to the maternal surface with a zone of infarction. Postdelivery, her hemoglobin rose to $8.7 \mathrm{gm} \%$, platelet count was 1.09 lacs $/ \mu \mathrm{L}$, and total leukocyte count was $35,500 /$ $\mu \mathrm{L}$. Patient was started on triple antibiotics, oral iron, and multivitamins postdelivery and was discharged in a stable condition after giving necessary preconception advice for future pregnancy.

\section{DISCUSSION}

Placental abruption is one of the serious complications of pregnancy, because it leads to both poor maternal and fetal outcome. The diagnosis is mainly clinical; however, laboratory and imaging findings can be used to support the clinical diagnosis. The immediate cause of premature placenta detachment is rupture of maternal vessels in the decidua basalis, which interact with the anchoring villi in the placenta. The etiology of bleeding in the decidua basalis remains speculative in most cases except in a small percentage of cases, where it is related to sudden mechanical events, such as blunt abdominal trauma or rapid uterine decompression. However, most causes appear to be related to some placental chronic disease. ${ }^{3,4}$ Usually, the cases of abruption present after 20 weeks of gestation with symptoms of vaginal bleeding ranging from mild to massive, abdominal pain and sometimes, features of maternal shock due to massive hemorrhage leading to disseminated intravascular coagulopathy, acute renal failure, and even maternal mortality. It is rare for abruption to occur before 20 weeks of gestation. However, the knowledge that this can occur even in early second trimester will help clinicians to make a timely diagnosis and manage the condition accordingly.

In a case report by Mellisa Walker et $\mathrm{al}_{1}^{5}$ a 30 -year-old women presented at 30 weeks of gestation with asymptomatic preeclampsia and her routine ultrasound revealed a large retroplacental sonolucent area of $4.7 \times 1.6 \mathrm{~cm}$. She was admitted, kept on continuous monitoring, steroids were given, and cesarean section was done in view of increasing size of retroplacental clot of $6.3 \times 2.2 \mathrm{~cm}$. Placental pathology revealed chronic abruption. It was suggested that timely diagnosis and intervention led to favorable maternal and fetal outcome.

Elliott et $\mathrm{al}^{6}$ did a retrospective study including all patients with a diagnosis of placental abruption with oligohydramnios or ruptured membranes defined as CAOS. Twenty-four cases with CAOS were identified. Out of these, 14 patients had evidence of abruption at less than 20 weeks. The mean gestational age at first bleeding was $19.4 \pm 5.5$ weeks, with the mean gestational age at delivery being $28.1 \pm 4.5$ weeks. Preterm rupture occurred in 15 out of 24 cases.

\section{CONCLUSION}

It is important to distinguish placental abruption as one of the causes of bleeding and pain abdomen during the early phase of the second trimester so that timely diagnosis and intervention can lead to better patient management and decrease in maternal morbidity. Hence, our report demonstrates the importance of the clinical examination associated with obstetric ultrasonography in diagnosis and aggressive management for a better maternal outcome.

\section{CLINICAL SIGNIFICANCE}

Chronic abruption may present with vague clinical features, especially in early pregnancy, but a high index of suspicion should be kept in mind for timely recognition and management.

\section{REFERENCES}

1. Tikkanen M. Placental abruption: epidemiology, risk factors and consequences. Acta Obstet Gynecol Scand 2011 Feb;90(2):140-149.

2. Oyelese $Y$, Ananth CV. Placental abruption. Obstet Gynecol 2006 Oct;108(4):1005-1016.

3. Ananth CV, Oyelese Y, Yeo L, Pradhan A, Vintzileos AM. Placental abruption in the United States, 1979 through 2001: 
temporal trends and potential determinants. Am J Obstet Gynecol 2005 Jan;192(1):191-198.

4. Ananth CV, Getahun D, Peltier MR, Smulian JC. Placental abruption in term and preterm gestations: evidence for heterogeneity in clinical pathways. Obstet Gynecol 2006 Apr;107(4):785-792.
5. Walker M, Whittle W, Keating S, Kingdom J. Sonographic diagnosis of chronic abrption. J Obstet Gynaecol Can 2010 Nov;32(11):1056-1058.

6. Elliott JP, Gilpin B, Strong TH Jr, Finberg HJ. Chronic abruption oligohydramnios sequence. J Reprod Med 1998 May;43(5): 418-422. 